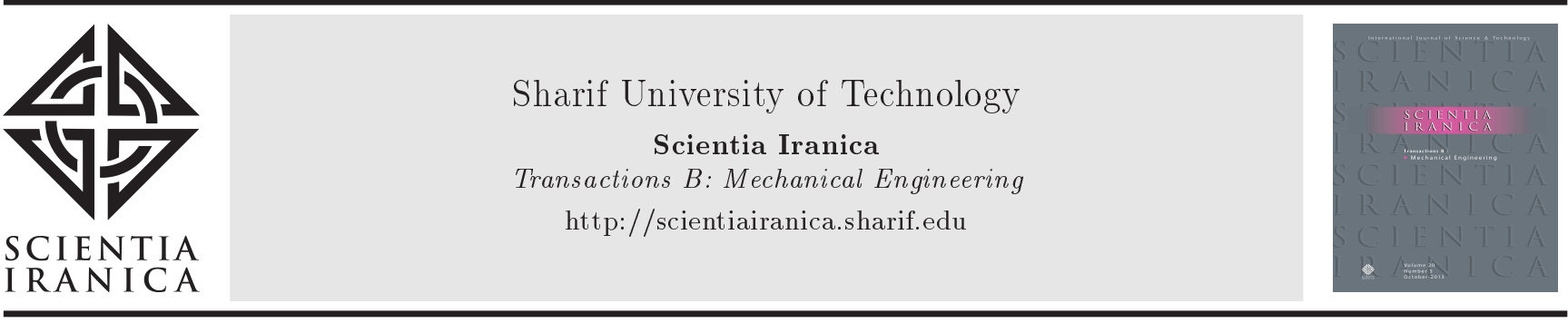

\title{
Post-fatigue life prediction of glare subjected to low-velocity impact
}

\author{
A. Sedaghat, M. Alitavoli*, A. Darvizeh, and R. Ansari Khalkhali \\ Faculty of Mechanical Engineering, University of Guilan, Rasht, Iran.
}

Received 12 May 2019; received in revised form 16 September 2019; accepted 12 October 2020

\section{KEYWORDS}

GLARE;

Fatigue life;

Multilayer neural

network;

Genetic algorithm.

\begin{abstract}
This study first modeled the dynamic of progressive failure of glass-fiberreinforced aluminum laminates (GLARE) under low energy impact using intra laminar damage models based on strain-based damage evolution laws, Puck failure criteria, and ABAQUS-VUMAT. For delamination of interface and aluminum layers, the bilinear cohesive model and the Johnson-Cook model were employed, respectively. The fatigue life of the fiber metal laminates of GLARE subjected to impact was obtained and the numerical and experimental results of the model were compared with each other. By considering the very good match between the numerical and experimental results, the results of the finite element model were generalized and expanded and following the use of the multilayer neural network, the numerical model was extracted. Then, by applying the metainnovative algorithm, the maximum fatigue life of GLARE was determined at the highest level with very low-velocity impact; the best configuration of three-layer GLARE was selected. The findings indicated that the best configuration of hybrid composite GLARE based on conventional commercial laminates that could tolerate low-velocity impacts with 18J impact energy and a $349 \mathrm{MPa}$ fatigue load with a frequency of $10 \mathrm{~Hz}$ was [Al/0-90-900/Al/0-90-0/Al/0-90-90-0/Al] with a cycle life time of 13016 .

(C) 2021 Sharif University of Technology. All rights reserved.
\end{abstract}

\section{Introduction}

Glass-fiber-reinforced aluminum laminates (GLARE) belongs to a family of fiber-metal laminates composed of alternate layers of prefabricated reinforced composites (prepreg) with unidimensional glass fibers and aluminum 2024 sheets, first invented for aeronautical applications [1]. Today, GLAREs are presented with 6 different standard classes (Table 1), GLARE 1 to GLARE 6. Figure 1 shows a general view of the GLARE.

\footnotetext{
*. Corresponding author.

E-mail addresses: sedaghat.alirezaa@gmail.com (A. Sedaghat); tavoli@guilan.ac.ir (M. Alitavoli);

adarvizeh@guilan.ac.ir (A. Darvizeh); r_ansari@guilan.ac.ir (R. Ansari Khalkhali)
}

doi: $10.24200 /$ sci. 2019.53550 .3297
Among glass fibers, GLARE has good adhesive properties. Glass fibers are characterized by many more strengths than compressive loading. For this reason, failure of glass fibers is rarely observed during fatigue loading. Besides, it is attributed with significant advantages including high compressive and tensile strength, good impact behavior, and high residual strength [2]. Good adhesion between resin and glass fibers in GLARE has made the fabrication of fibers on both sides possible. Accordingly, GLARE is suitable for structures subjected to bilateral stresses. It appears that the characteristics of GLARE make it eligible for various potential applications and they include weight saving, good impact resistance, and damage tolerance [3-5]. Impact-induced damage is one of the important issues in aerial structures. The inability to detect internal damages of composite layers earlier, which sometimes originates from the area with damages, is 
Table 1. Commercial grades of glass-fiber-reinforced aluminum laminates (GLARE).

\begin{tabular}{cccccc}
\hline Grade & Sub & Metal type & $\begin{array}{c}\text { Metal thickness } \\
(\mathbf{m m})\end{array}$ & $\begin{array}{c}\text { Fibre layer } \\
(\mathbf{m m})\end{array}$ & $\begin{array}{c}\text { Pregreg orientation } \\
\text { in each fibre } \\
\text { layer }\left(^{\circ}\right)\end{array}$ \\
\hline GLARE 1 & - & $7475-\mathrm{T} 761$ & $0.3-0.4$ & 0.266 & $0 / 0$ \\
GLARE 2 & GLARE 2A & $2024-\mathrm{T} 3$ & $0.2-0.5$ & 0.266 & $0 / 0$ \\
GLARE 3 & GLARE 2B & $2024-\mathrm{T} 3$ & $0.2-05$ & 0.266 & $90 / 90$ \\
GLARE 4 & GLARE 4A & $2024-\mathrm{T} 3$ & $0.2-0.5$ & 0.266 & $0 / 90$ \\
& GLARE 4B & $2024-\mathrm{T} 3$ & $0.2-0.5$ & 0.266 & $0 / 90 / 0$ \\
GLARE 5 & - & $2024-\mathrm{T} 3$ & $0.2-0.5$ & 0.266 & $90 / 0 / 90$ \\
GLARE 6 & GLARE 6A & $2024-\mathrm{T} 3$ & $0.2-0.5$ & 0.266 & $0 / 90 / 90 / 0$ \\
& LARE 6B & $2024-\mathrm{T} 3$ & $0.2-0.5$ & 0.266 & $+45 /-45$ \\
\hline
\end{tabular}

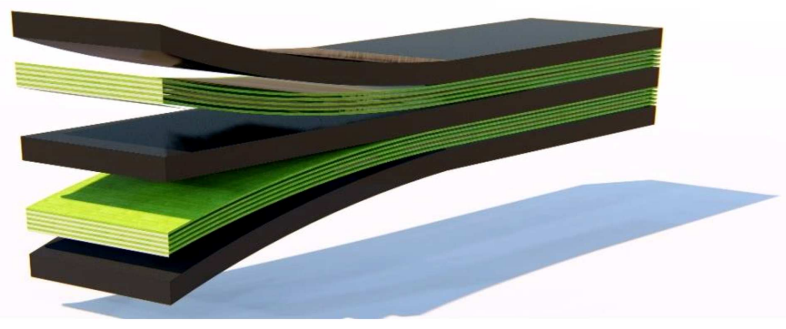

Figure 1. Fiber metal laminate glass-fiber-reinforced aluminum laminates (GLARE).

still a serious safety issue. Therefore, an accurate prediction of the internal impact damage is quite necessary. Generally, failure mechanics and fracture mechanics are respectively employed to simulate the failure of plates and model the post-impact damages to plates. These two theories have been combined with stall measure and plasticity to analyze non-linear behavior. In continuous failure mechanics, every consistent equation for damaged materials can somehow be similar to intact materials except the conventional stress, which has been replaced by effective stress [6,7].

Fracture mechanics can predict the initiation and growth of delamination phenomenon based on the overall energy release rate. Although there are general frameworks at play here, no general model for simulating the impact mechanics of GLARE has been suggested yet. There are a number of numerical simulations in this specific field. A suitable impact model can be developed for GLARE only if the basic role of constitutive materials in the impact reaction and the energy-absorbance feature is determined. Liao and Liu [8] investigated the absorbed energy among the constitutive materials of GLARE under low-velocity impact.

Fatigue crack growth in GLAREs can be classified into two main mechanisms: crack growth on metal layers and delamination of the fiber-metal interface. In fact, these two share a balanced mechanism called coupling process. Fatigue crack growth in GLAREs can be described by Linear Elastic Fracture Mechanics (LEFM). This theory states that like metals, the crack growth rate of GLAREs corresponds to the stress concentration factor of the crack tip; however, this influence is not so simple because the above factor in GLAREs is itself influenced by bridging of fibers which initiates due to the delamination of the metal-fiber interface. When cracks on metal layers start growing, fibers remain intact over the crack length. These fibers create a load transfer pass over the crack and restrain crack opening. Therefore, a force with lower degree is transferred around the crack tip on metal layers which, in turn, reduces the stress concentration factor of the crack tip [9-18]. By considering the present metaheuristic algorithms, the multilayer perceptron neural network was selected for modeling due to their high performance in educability and the possibility of approximating non-linear results. Genetic algorithm is a general method for optimization and it benefits from genetic evolution for problem solving. The input is the problem that should be solved, and the corresponding solutions are codes based on a certain pattern and a metric is called fitness function, which randomly selects and evaluates every candidate solution. In the following, a short summary of the neural network and genetic algorithm is given [19-24].

\subsection{Multilayer neural network}

Back-Propagation (BP) algorithm is used for training feed-forward multilayer neural networks, commonly known as Multi-Layer Perceptron (MLP) networks. Error BP algorithm includes two main passes. The first pass is forward pass in which an input vector is provided to MLP network and its effects are propagated forward from the hidden layer to the output layer and the output layer presented in the output layer creates a real response from the MLP network. The second pass is called backward in which unlike the forward pass, 


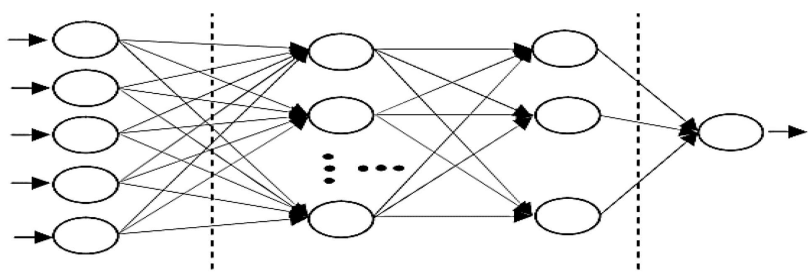

Figure 2. Neural network model used in this study [21].

the parameters of the MLP network can be changed and adjusted. This adjustment is conducted according to the error correction law and the error signal is created in the output layer of the networks. The error vector is the difference between the desired and real responses coming from the network. The calculated error value is distributed over the whole network in the backward pass through the network layers. Given that this distribution is against the communication pass for synapse weights, the term "error BP" has been used for this algorithm. One of the problems that remains challenging to researchers is the overconsistency of neural networks in the training process. In other words, the training error level is quite low in networks; however, the error level is very high for test data and the network preserves training data samples; therefore, it does not have the generalizability power for new data. To solve this problem, data are divided into three sets: training, validation, and testing [19]. In the remaining part of the algorithm, the network parameters are arranged such that the real response from the network be as close as possible to the desired response [20] (Figure 2).

\subsection{Optimizations with genetic algorithm}

In most of the optimization problems in engineering, optimization of more than one target function is of great importance for designers and also, there are a number of general inconsistent target functions that should be optimized simultaneously by the designer. In contrast to single-objective problems which imply the existence of only one extremum for the problem, a set of design vectors is obtained for these problems as solutions called Pareto; in this respect, the designer selects one of these points on demand. Another solution is using highest probability decoding display Approach [21-23].

Genetic algorithm considers every optimization problem as evolutionary. This algorithm selects a set of possible values so as to find the optimum ones for parameters. Then, by applying the evolution process over this population, each parameter gradually undergoes changes so as to reach an optimum value. Genetic algorithm needs a primary population at first. The primary population evolves through genetic changes in their chromosomes such that following the creation of new generations, a solution to the optimization problem can be gradually found. This algorithm simulates three main operators to evolve the population including mutation, crossover, and selection.

In the crossover, a child chromosome is produced through the breeding of two parent chromosomes such that only some genes of the two selected chromosomes could be allocated rather than each available gene. The crossover methods include single-point and multipoint crossovers. These crossover points are created using random numbers. In the mutation, some of the randomly selected genes undergo random changes. For example, in binary encoding, zeroes might be changed to one, and vice versa.

In this way, the primary population varies and a new population can be produced. This new population is created by children. In the selection process, one set of chromosomes is chosen from their previous population based on their fitness with random numbers. The fittest chromosome stands a much better chance of surviving in the next generation. The selected population takes the position of its parents. There are many different methods for selection. In this study, a fortune wheel method was used for the genetic algorithm. All crossover, mutation, fitness, and selection stages are common among all genetic algorithms. The procedure for performing the genetic algorithm is given in Figure 3.

For multi-objective optimization problem-solving, various methods exist each of which is characterized by certain strengths and weaknesses. Weight coefficient method, which is one of the least complicated methods for multi-objective optimization problem-solving, was used in this study. In this method, the target functions of the problem were combined with each other through

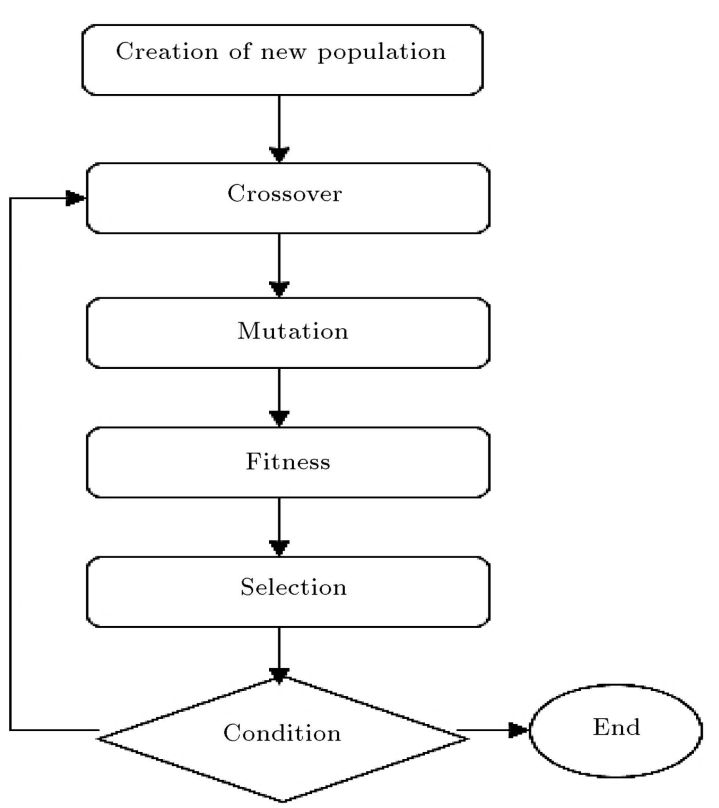

Figure 3. Procedure for the genetic algorithm [20]. 
their weight coefficients and the final target function was produced. The value of the weight coefficient of every target function was dependent on its importance, i.e., more important target functions received larger weight coefficients $[23,24]$. In this method, a multiobjective optimization problem was turned to a singleobjective one.

In addition, a major issue is that the obtained solution point according to the values of weight coefficient is one of Pareto chart's points. Therefore, by changing the weight coefficients, one point of the Pareto chart could be found in each perform [24].

\section{Materials and methods}

In the present investigation, the post-impact fatigue life of GALRE was analyzed using the simulation of impact test and tension-tension fatigue test; then, a new model was developed based on the good consistency between the numerical and experimental results. Then, the obtained models by Abaqus software were used in a four-layer perceptron neural network and a numerical model for its results was obtained. Next, the numerical results of the neural network were used for optimization of the genetic algorithm in order to obtain a three-layer arrangement for GLARE to reach the maximum fatigue life upon the highest-level low-velocity impact. In the following, the experimental methods are described orderly.

\subsection{Numerical method for GLARE under low-energy impact}

For predicting the mechanical behavior of the aluminum layer, the Johnson-Cook relation without temperature consideration was used [25]:

$$
\sigma=\left[A+B\left(\bar{\varepsilon}_{p l}\right)^{n}\right]\left[1+C \ln \frac{\dot{\bar{\varepsilon}}_{p l}}{\dot{\varepsilon}_{0}}\right]
$$

where $A, B$, and $C$ are material parameters, $\dot{\bar{\varepsilon}}_{p l}$ is an equivalent plastic strain, and $n$ is material constant. A damage parameter $\Psi$ was used for simulation of the ductile damage of the aluminum layer.

$$
\begin{aligned}
& \psi=\sum\left(\frac{\Delta \bar{\varepsilon}_{p l}}{\bar{\varepsilon}_{f}^{p l}}\right), \\
& \bar{\varepsilon}_{f}^{p l}=\left[d_{1}+d_{2} \exp \left(d_{3} p / q\right)\right]\left[1+d_{4} \ln \frac{\dot{\varepsilon}_{p l}}{\dot{\varepsilon}_{\circ}}\right],
\end{aligned}
$$

where $d_{1}$ to $d_{4}$ are material parameters and $p / q$ is the hydrostatic pressure per deviatoric stress.

\subsection{Composite damage model}

The intra-laminar damage model enjoys the ability to prognosticate the intralaminar failure of a composite material the results of which were obtained by Donadon et al. [26] and Appruzzese and Falzon [27]. Damage variables represent the amount of local damage in a Representative Volume Element (RVE) of the composite material. In one dimension, the definition of damage variable $d$ leads to the effective stress $\bar{\sigma}$ or the stress computed over the section of the RVE. For an undamaged pristine material, $d=0 ; d=1$ denotes the complete failure. Lemaitre and Chaboche expressed that the constitutive law of damaged material could be derived from the principle of strain equivalence.

The relationship existing between the stress tensor $\bar{\sigma}$ and the true stress tensor $\sigma$ is given below:

$$
\sigma=D \bar{\sigma},
$$

where $D$ is the damage matrix and is obtained by Eq. (5) as shown in Box I. The stress tensor $\bar{\sigma}$ and strain tensor $\varepsilon$ form a relation as given below:

$$
\bar{\sigma}=C \varepsilon .
$$

The relationship between the stress increment tensor $\Delta \sigma$ and strain tensor $\Delta \varepsilon$ is as follows:

$$
\Delta \sigma=C\left[D\left\{\Delta \varepsilon-\Delta \varepsilon^{i n}\right\}-\Delta D\left\{\varepsilon-\varepsilon^{i n}\right\}\right],
$$

where $\varepsilon^{\text {in }}$ is an inelastic strain and $D$ and $\Delta D$ are the damage and incremental damage matrices expressed by Donadon et al. [26].

\subsection{Material properties}

For simulating the finite element model with intralaminar and interlaminar damages together with a wide range of lamina properties, the material behavior should be described first. Tables 2 and 3 show all of the material properties used in this research.

\subsection{Finite element modeling}

Numerical modeling was conducted by ABAQUSVUMAT to evaluate the impact-induced damage of GLARE specimens. A C3D8R solid hexagonal element was used. The Johnson-Cook model was implemented for aluminum layers. The dynamic penalty method was applied to simulate the contact between the GLARE

\begin{tabular}{|c|c|}
\hline Elastic parameters & $\begin{array}{l}\rho=2770 \mathrm{~kg} / \mathrm{m}^{3}, E=72.4 \mathrm{GPa} . \\
\nu=0.34\end{array}$ \\
\hline Plastic parameters & $\begin{array}{l}A=396 \mathrm{MPa}, B=689 \mathrm{MPa} \\
C=0.083 \mathrm{MPa}, n=0.34\end{array}$ \\
\hline Fracture parameters & $\begin{array}{l}d_{1}=0.13, d_{2}=0.13, d_{3}=-1.5 \\
d_{4}=0.011, \dot{\varepsilon}_{0}=1.0 s^{-1}\end{array}$ \\
\hline
\end{tabular}
and impactor. Due to the excessive distortion of elements, enhanced stiffness relaxation was employed to control hourglassing phenomenon. Intralaminar

Table 2. The material parameters for 2024-T3 aluminum [8]. 


$$
D=\left\{\begin{array}{cccccc}
1 /\left(1-d_{11}\right) & \circ & \circ & \circ & \circ & \circ \\
\circ & 1 /\left(1-d_{22}\right) & \circ & \circ & \circ & \circ \\
\circ & \circ & 1 & \circ & \circ & \circ \\
\circ & \circ & \circ & 1 /\left(1-d_{12}\right) & \circ & \circ \\
\circ & \circ & \circ & \circ & 1 /\left(1-d_{23}\right) & \circ \\
0 & \circ & \circ & \circ & \circ & 1 /\left(1-d_{31}\right)
\end{array}\right\} .
$$

Box I

Table 3. Material parameters for glass/epoxy composite layer [8-10].

Young's moduli $E_{1}=50.6 \mathrm{GPa}, E_{2}=E_{3}=9.9 \mathrm{GPa}$
Shear moduli $G_{12}=G_{13}=3.7 \mathrm{GPa}$,
$G_{23}=1.65 \mathrm{GPa}$
Poisson's ratios
$\nu_{12}=\nu_{13}=0.32, \nu_{23}=0.063$
Strength parameters
Composite layer $\quad X^{T}=2500 \mathrm{MPa}, X^{C}=2000 \mathrm{MPa}, Y^{T}=50 \mathrm{MPa}$
$Y^{C}=150 \mathrm{MPa}, S_{12}=S_{13}=75 \mathrm{MPa}$
$S_{23}=50 \mathrm{MPa}[25]$
Intralaminar fracture toughness
$G_{11}^{T}=81.5 \mathrm{~N} / \mathrm{mm}, G_{22}^{C}=14.955 \mathrm{~N} / \mathrm{mm}[23]$
$G_{11}^{C}=106.3 \mathrm{~N} / \mathrm{mm}, G_{22}^{T}=14.955 \mathrm{~N} / \mathrm{mm}$,
Interlaminar fracture toughness
$G_{1}^{c}=0.5 \mathrm{~N} / \mathrm{mm}, G_{2}^{c}=G_{3}^{c}=0.9 \mathrm{~N} / \mathrm{mm}$

damage models with Puck failure criteria were implemented. The bilinear cohesive model was used to predict the delamination and the finite-thickness cohesive element was established for the interface delamination between the two layers.

\subsection{Fatigue crack growth in Fiber Metal Laminates (FMLs)}

Mechanism of fatigue crack growth in Fiber Metal Laminates (FMLs) has been a complex phenomenon. To characterize this phenomenon and develop an analytical method, several simplified assumptions were required. However, the suggested method according to the real crack growth phenomenon should still be accurate. The Stress Intensity Factor (SIF) at crack tip is expressed as follows:

$$
K_{\text {tip }}=K_{\text {farfield }}+K_{\text {bridging }} \text {. }
$$

The SIF expression, caused by the far-field stress applied to the Al layers, by the linear elastic theory for monolithic metals is as follows [13]:

$$
K_{\text {farfield }}=S_{a l} \sqrt{\pi a} .
$$

\subsection{Bridging stress calculation}

The fibers in FMLs were insensitive to fatigue. They transferred a significant portion of the load over the crack and restrained the crack opening, as shown in Figure 4. Due to this restraining, the crack opening in GLARE was smaller than the monolithic metal. This mechanism resulted in smaller crack at tip SIF than the monolithic metal for equal crack length and applied load (Figure 4).

The crack opening of a fatigue crack in infinite FMLs at location $x$ along the crack could be expressed as follows:

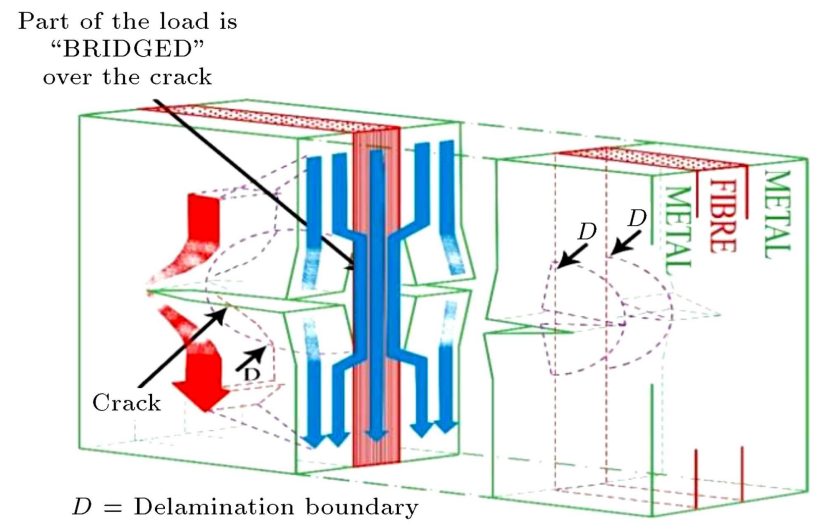

Figure 4. Crack bridging between the fibers and delamination of the layers. 


$$
v_{\infty}(x)-v_{b r}(x)=\delta_{f}(x)+\delta_{p p}(x),
$$

where $v_{\infty}(x), v_{b r}(x), \delta_{f}(x)$, and $\delta_{p p}(x)$ represent the crack opening displacement caused by far-field stress, bridging stress in the aluminum layer, elongation of the fibers over the delaminated length, and deformation of the glass/epoxy prepreg layer, respectively. Crack opening displacement far from the crack caused by the far-field stress is obtained as follows:

$$
v_{\infty}(x)=2 \frac{S_{a l}}{E_{a l}} \sqrt{a^{2}-x^{2}} .
$$

$E_{a l}, S_{a l}$, and $a$ are the elastic modulus of aluminum, far-field stress of aluminum layer, and crack length, respectively.

Heretofore, the SIF at the crack tip for a crack with an arbitrary delamination shape was determined and the crack growth rate could be obtained through the empirical Paris relation [28] for the aluminum layer:

$$
\begin{aligned}
& \frac{d a}{d N}=C_{c g} \Delta K_{e f f}^{n_{e g}}, \\
& \Delta K_{e f f}=\left(1-R^{1 / 35}\right) K_{t i p}^{\prime} \sqrt{\sec \left(\frac{a \pi}{W}\right)}, \\
& K_{\text {tip }}^{\prime}=\beta_{\text {dixon }} \times \beta_{\text {impact }} \times K_{\text {tip }},
\end{aligned}
$$

where:

$$
\begin{aligned}
& \beta_{\text {dixon }}=\frac{1}{\sqrt{1-\left(\frac{2 a}{W}\right)^{2}},} \\
& \beta_{\text {impact }}=\frac{1}{\sqrt{1-\left(\frac{2 v_{\text {impact }}}{v_{\text {ballistic }}}\right)^{2}}},
\end{aligned}
$$

where $v_{\text {impact }}$ and $b_{\text {allistic }}$ are impactor velocity and ballistic limit of GLARE types, which were investigated by Seyed Yaghobi and Liaw [29]. Crack growth life of GLARE could be calculated through Eq. (4):

$$
N=\int_{a_{\circ}}^{a} \frac{1}{C_{c g} \Delta K_{e f f}^{n_{c g}}} d a .
$$

\subsection{Specimens}

For GLAREs 52.1 and $43 / 2$, the thickness of aluminum and composite layers was $0.4 \mathrm{~mm}$ and $2.2 \mathrm{~mm}$, respectively, with the total thickness of $3 \mathrm{~mm}$.

\subsection{Low-velocity impact testing}

Impact testing was conducted by a drop-weight impact tower in the velocity range of $1.87-2.39 \mathrm{~m} / \mathrm{s}$ (Figures 5 and 6 ). After the first impact, a pneumatic braking system was activated and the impactor was prevented from hitting the specimen again. The specimen was clamped between the top and bottom steel plates of the fixture with $75 \times 225 \mathrm{~mm}^{2}$ with a $50 \mathrm{~mm}$ diameter circular opening at the center of each plate. The impactor was a hemispherical steel with a diameter of $10 \mathrm{~mm}$ and a mass of $6.29 \mathrm{~kg}$.

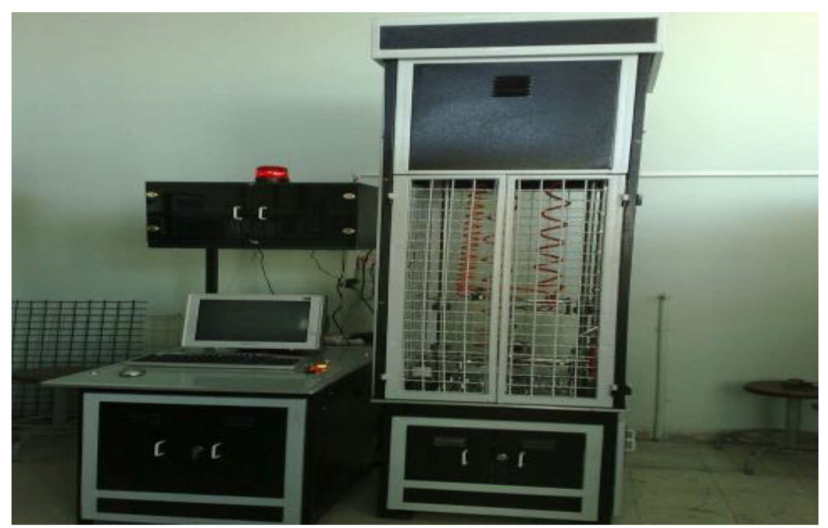

Figure 5. The impact test machine.

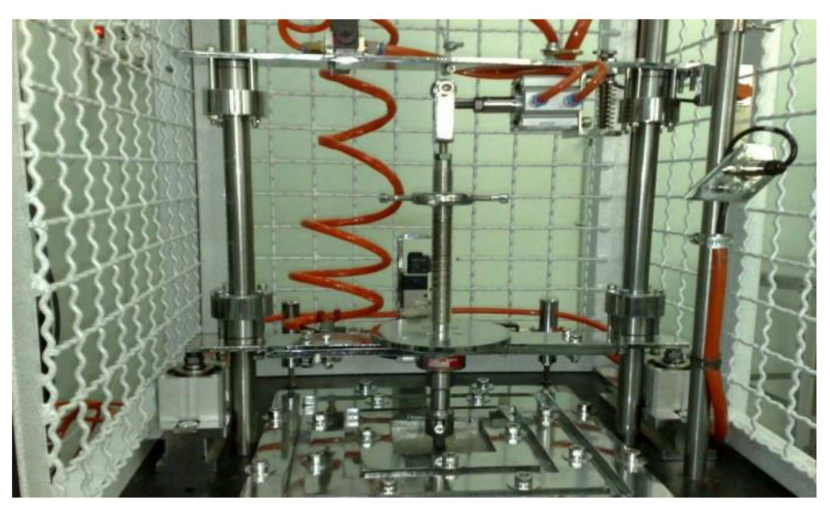

Figure 6. Impact loading setup.

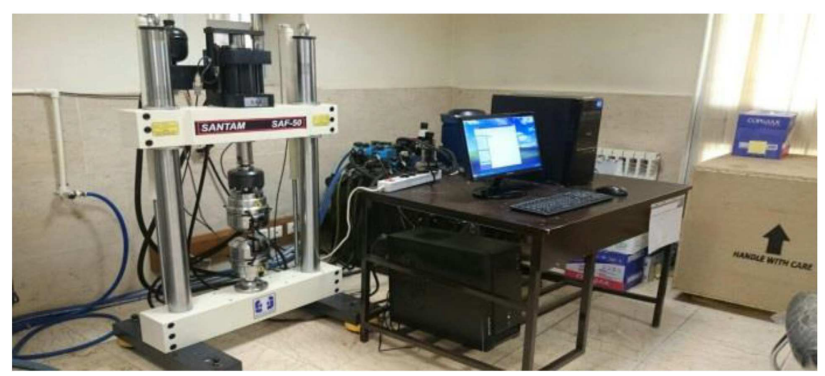

Figure 7. The fatigue test machine.

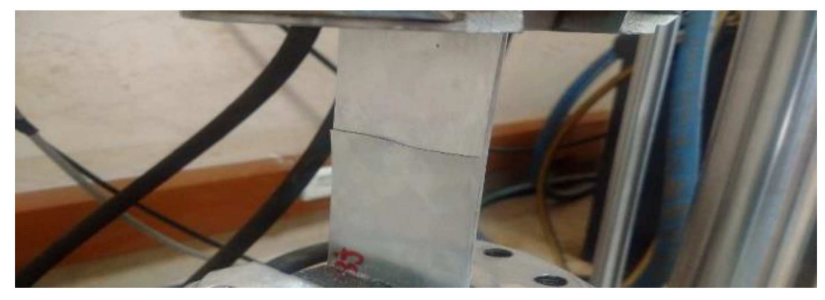

Figure 8. Fatigue test specimen.

\subsection{Post-impact fatigue testing}

The tension-tension fatigue tests were conducted by a SANTAM-50 machine at 5 levels of 175, 232, 291, and $349 \mathrm{MPa}$ with the stress ratio of $R=0.05$ and a frequency of $10 \mathrm{~Hz}$ (Figures 7 and 8). 
Table 4. The results of finite element simulation in creating a numerical model.

\begin{tabular}{|c|c|c|c|c|c|c|c|}
\hline Lifetime & Third layer & Second layer & First layer & Lifetime & Third layer & Second layer & First layer \\
\hline 6700 & 3 & 2 & 1 & 7900 & 1 & 0 & 1 \\
\hline 8900 & 1 & 3 & 2 & 7000 & 2 & 2 & 2 \\
\hline 8000 & 4 & 1 & 3 & 8400 & 3 & 4 & 3 \\
\hline 8000 & 1 & 2 & 4 & 8000 & 4 & 1 & 4 \\
\hline 7200 & 3 & 4 & 0 & 9300 & 0 & 2 & 1 \\
\hline 6800 & 2 & 1 & 1 & 8600 & 3 & 4 & 2 \\
\hline 9200 & 4 & 3 & 2 & 8300 & 2 & 1 & 3 \\
\hline 5600 & 0 & 4 & 3 & 8300 & 4 & 3 & 4 \\
\hline 8200 & 3 & 0 & 4 & 11000 & 1 & 5 & 5 \\
\hline 6000 & 2 & 2 & 0 & 5300 & 4 & 0 & 1 \\
\hline 7600 & 4 & 4 & 1 & 8200 & 2 & 2 & 2 \\
\hline 9000 & 1 & 1 & 2 & 8000 & 5 & 4 & 3 \\
\hline 6400 & 3 & 2 & 3 & 8300 & 0 & 1 & 4 \\
\hline 11300 & 2 & 4 & 4 & 10900 & 3 & 3 & 5 \\
\hline 8000 & 5 & 1 & 1 & 4900 & 1 & 4 & 0 \\
\hline 7700 & 0 & 3 & 2 & 6800 & 4 & 2 & 1 \\
\hline 10000 & 3 & 5 & 3 & 8900 & 1 & 3 & 2 \\
\hline 8400 & 1 & 0 & 4 & 10000 & 3 & 5 & 3 \\
\hline 9200 & 4 & 2 & 5 & 7600 & 2 & 0 & 4 \\
\hline 5200 & 1 & 4 & 1 & 6100 & 5 & 2 & 0 \\
\hline 7800 & 3 & 1 & 2 & 8200 & 1 & 3 & 1 \\
\hline 9200 & 2 & 3 & 3 & 7800 & 3 & 1 & 2 \\
\hline 10000 & 5 & 4 & 4 & 7300 & 2 & 2 & 3 \\
\hline 7800 & 0 & 1 & 5 & 11900 & 4 & 4 & 4 \\
\hline 9100 & 3 & 3 & 0 & 6900 & 1 & 1 & 0 \\
\hline 8000 & 2 & 5 & 1 & 9000 & 4 & 3 & 1 \\
\hline 7900 & 4 & 0 & 2 & 8100 & 2 & 5 & 2 \\
\hline 8800 & 1 & 2 & 3 & 9000 & 5 & 0 & 3 \\
\hline 8300 & 4 & 3 & 4 & 9400 & 0 & 2 & 4 \\
\hline 6200 & 2 & 1 & 0 & & & & \\
\hline 7900 & 5 & 2 & 1 & & & & \\
\hline 4700 & 0 & 4 & 2 & & & & \\
\hline 7800 & 3 & 1 & 3 & & & & \\
\hline 10500 & 1 & 3 & 4 & & & & \\
\hline
\end{tabular}

\section{Findings}

A total of 64 cases were simulated using ABAQUS software, as listed in Table 4. Three variables were used for designation: the designs of different GLARE configurations. Every location could have one out of five GLARE types and zero values presented in the table, indicating the non-existence of any GLARE in that location. Taguchi method was used for model designing. Model designing was conducted with maximum impact energy and maximum imposed fatigue stress.

\section{Results and discussion}

\subsection{Evaluation of the finite element}

A set of low-velocity impact tests was conducted to evaluate deformation and distortion of GLAREs. Com- parative studies were conducted based on the results of experiments on GLARE 52.1 and $43 / 2$ for the evaluation of prediction models. A $50 \times 200 \mathrm{~mm}^{2}$ GLARE specimen was clamped between the top and bottom of the steel plates. A steel spherical impactor with a $10 \mathrm{~mm}$ diameter and a mass of $6.29 \mathrm{~kg}$ was used for $11 \mathrm{~J}$ and $18 \mathrm{~J}$ impact energies. A comparison between GLARE 5 2/1 and 4 3/2 using the prediction model for the contact time history showed that the obtained results were consistent with the experimental results, as represented in Figures 9-13.

According to Figure 9, based on the predicted pattern for this problem in which the stress concentration was higher in the encountered area, graded meshing was used to have a more accurate dynamic analysis of the impactor area with the GLARE surface. Following the collision of the impactor and GLARE, the force val- 


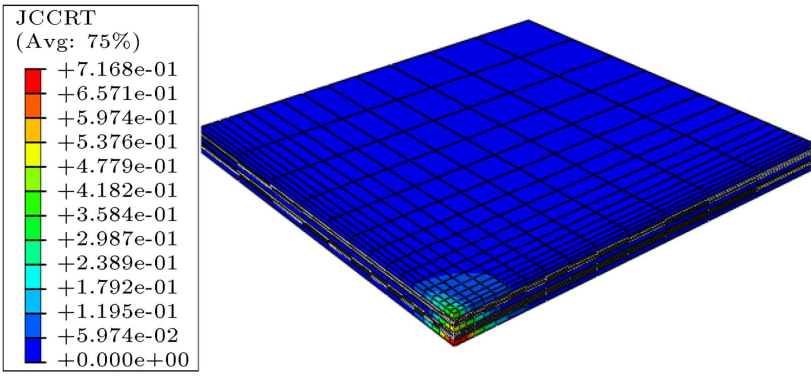

Figure 9. A complete view of the finite element modeling for glass-fiber-reinforced aluminum laminates (GLARE) 5 $2 / 1$.

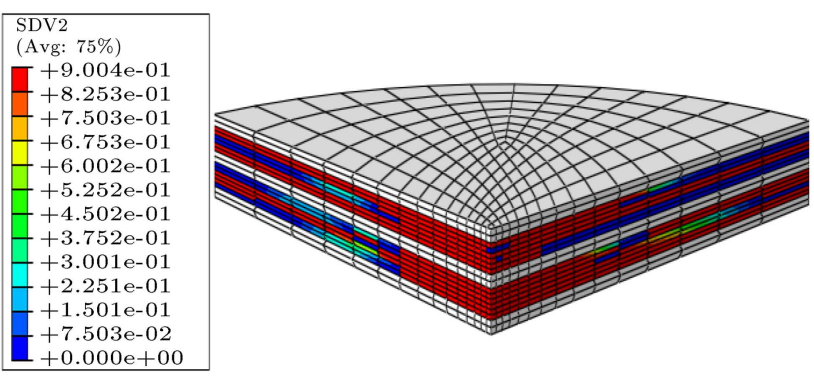

Figure 10. Damage cantor for the composite part of glass-fiber-reinforced aluminum laminates (GLARE).

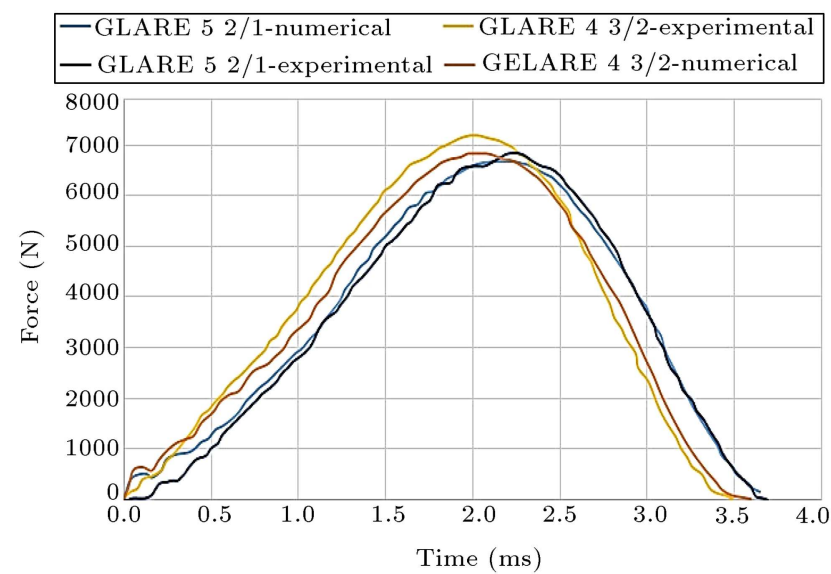

Figure 11. Comparison between numerical and experimental results of force-time history for glass-fiber-reinforced aluminum laminates (GLARE) 52.1 and $43 / 2$.

ues for central elements increased intensely and reached the maximum level. This maximum limit occurred when the GLARE 5 2/1 was at its extreme deflection and after that, the impactor started dissociating from GLARE surface and the force decreased down to zero, i.e., complete separation. Figure 11 shows the impact force-time history curves for GLAREs 5 2/1 and $43 / 2$ using $18 \mathrm{~J}$ impact energy. Some numerical oscillations appeared near the peak force in the curves, mainly because of the dynamic response and numerical effect. In the present investigation, a numerical filtering technique was applied and the output sampling time

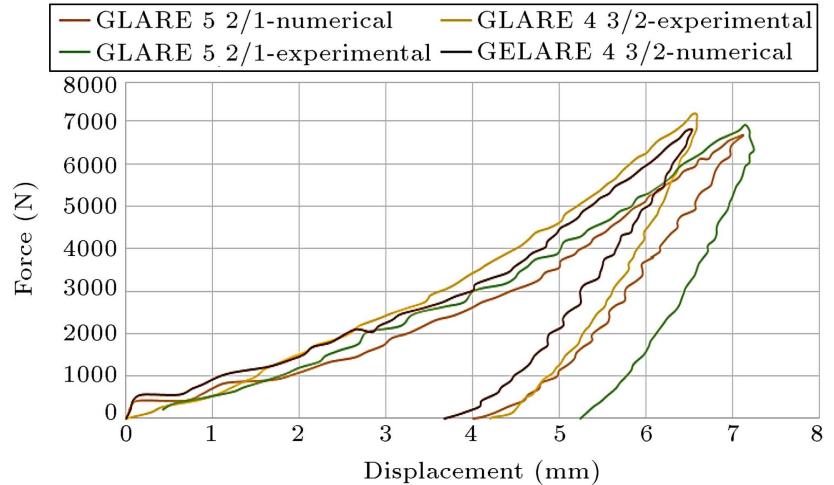

Figure 12. Force-displacement history curve for $18 \mathrm{~J}$ impact energy.

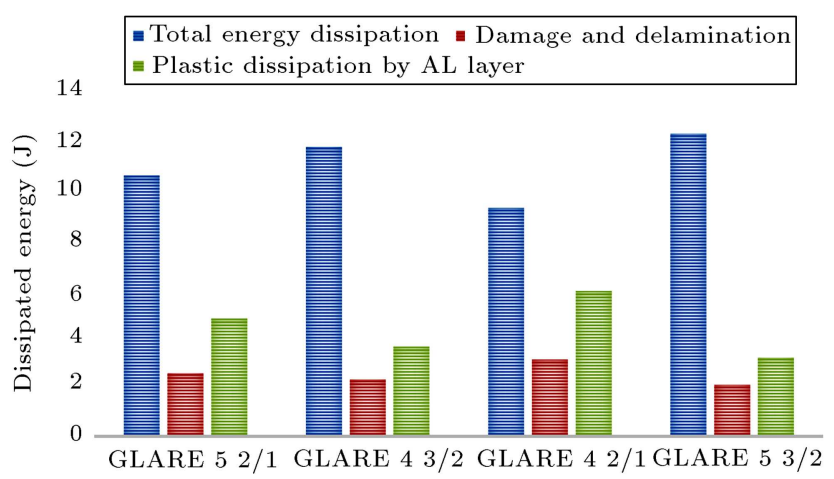

Figure 13. Results of post-impact fatigue for glass-fiber-reinforced aluminum laminates (GLARE) 52.1 .

was $0.005 \mathrm{~ms}$ at about $2 \mathrm{~ms}$ and $2.2 \mathrm{~ms}$ time for GLARE 5 2/1 and GLARE $43 / 2$ specimens, respectively. In addition, the impactor reached the lowest end and then, started to rebound until there was complete separation between the impactor and laminate at about $3.7 \mathrm{~ms}$ and $3.5 \mathrm{~ms}$ time, respectively. Relatively good agreement was found between the numerical and experimental results considering the impact force-time curves. For both numerical and experimental results of GLARE $43 / 2$ and GLARE 5 2/1, higher peak force and shorter contact time belonged to GLARE $43 / 2$. This was because of the number of aluminum layers characterized by higher stiffness and impact resistance. Figure 12 shows the maximum central displacement and permanent central displacement related to the lowest end of the impactor and the zero-impact force at the end of the impact, respectively, because GLARE $43 / 2$ absorbed much more energy due to the number of its aluminum and composite layers and lower displacement than GLARE 5 2/1. Therefore, it could be concluded that in response to a very slow velocity impact, with an increasing number of composite and aluminum layers in GLARE, more energy was absorbed and the displacement decreased.

Figure 13 shows the post-impact fatigue for GLARE 52.1 at different impact energy levels and 
different tension-tension fatigue tests. As the figure clearly shows, with an increase in the impact energy at a certain level of tensile stress in the fatigue test, the fatigue life of the specimen decreased due to the damage caused by the low-velocity impact. Also, in a condition characterized by high tension stress in fatigue and a constant energy level, the fatigue life of GLARE was reduced. The reason for this is that the damage level of the composite and aluminum layers of GLARE increased. Therefore, following the simultaneous increase of the impact on the GLARE and the tension stress level of fatigue, the fatigue life of GLARE was definitely reduced.

\subsection{The results of numerical modeling with neural network}

An MLP multilayer perceptron neural network was used for numerical modeling. Four layers with 8 hidden neurons produced a neural network. Of 64 primary datasets, $70 \%$ were used for training, $15 \%$ for testing, and $15 \%$ for validation; the improvement procedure is shown in Figure 10. Also, the difference between real outputs of the obtained neural network and 64 primary datasets is shown in Figure 14.

According to Figure 15, the blue points are fatigue lifetime of different GLAREs predicted by the neural network, while the red points are the fatigue lifetime modeled by the finite element method. As can be seen, these two methods were in agreement in terms of many points. Therefore, this figure clearly shows that the neural network can accurately predict the lifetime of GLARE specimen according to the primary data.

\subsection{Optimization}

Metaheuristic optimization was employed for the obtained models with a MLP neural network.

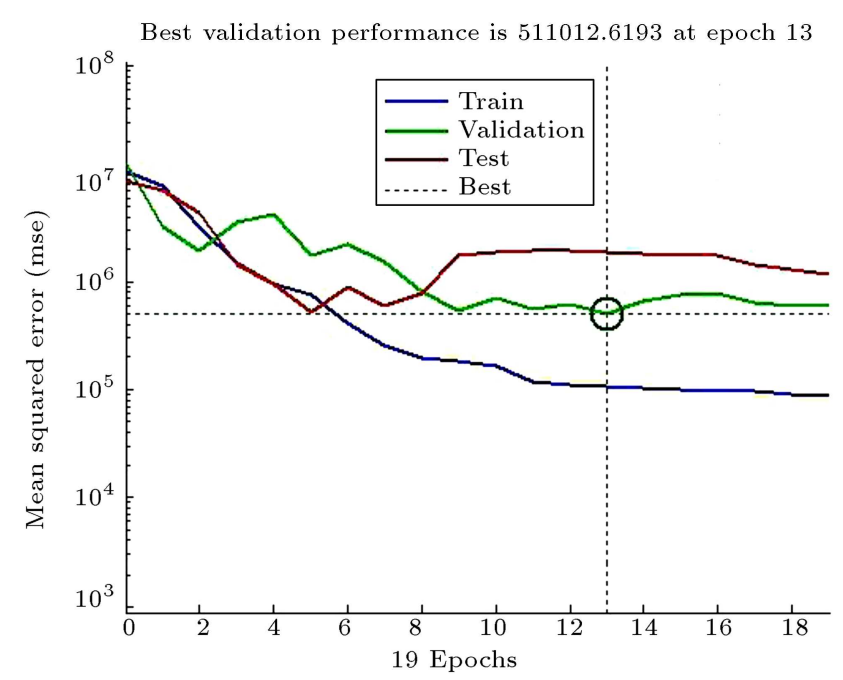

Figure 14. The improvement procedure of the neural network.

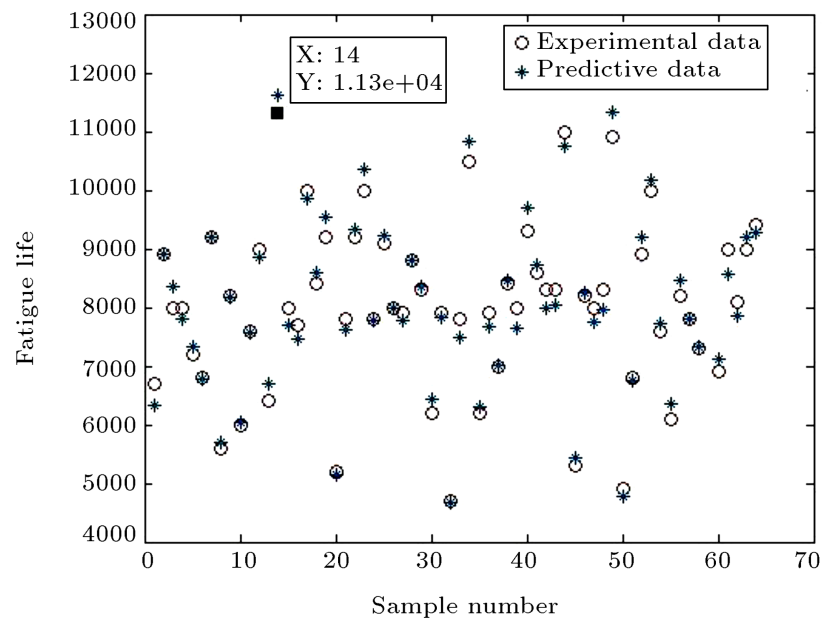

Figure 15. The difference between the real outputs of the obtained neural network.

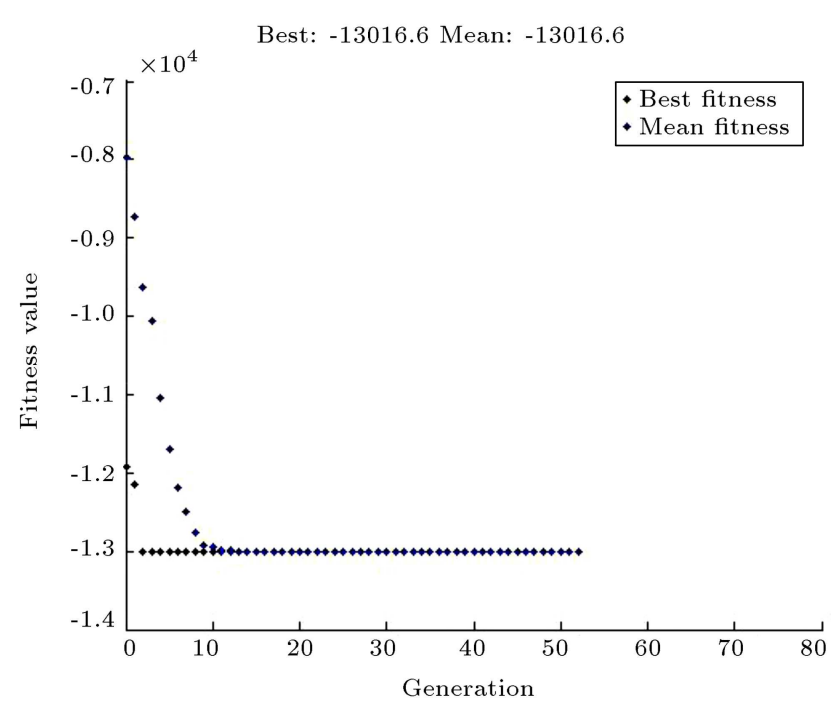

Figure 16. Convergence of the optimization results.

1- Design variables: permutation of different GLAREs;

2- Design constraints: 3 permutations and 5 different GLAREs;

3- Design objective: maximizing fatigue life.

Genetic algorithm was applied to these aims. The number of the primary population was 30 and the number of optimization generations was 80 . Due to the $85 \%$ crossover probability and a $15 \%$ mutation probability, the optimization procedure chart is shown in Figure 15. According to Figure 16, following the ninth generation of optimization, the two relative graphs of compatibility and optimization converged with each other and the compatibility was optimized after the tenth generation. Also, a lifetime of 13016 cycles was obtained for an impact energy of $18 \mathrm{~J}$ (the maximum of very low impact energy) and a $350 \mathrm{MPa}$ 


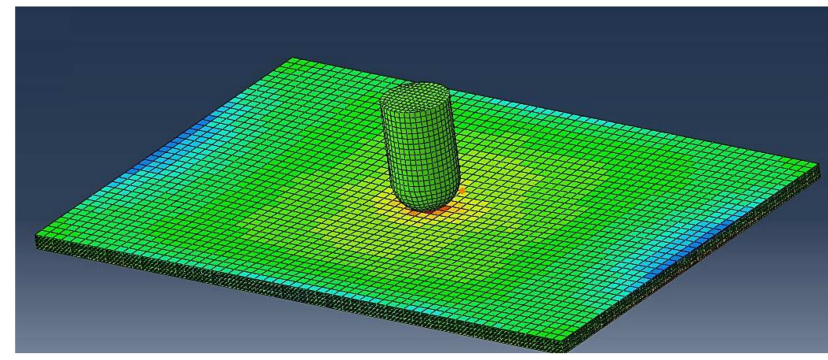

Figure 17. The finite element model for the new glass-fiber-reinforced aluminum laminates (GLARE).

tension-tension fatigue stress (the maximum exerted stress for tension-tension fatigue) for a GLARE with the first order equal to 5 , the second layer equal to 3 , and the third layer equal to 5 . It should be mentioned that GLARE 5 fatigue life in these loading conditions was 9873 cycles and the improvement rate was $31.83 \%$ compared to the new hybrid composite design. Moreover, compared to multilayer composite arrangements of [Glare 3/GLARE 5/ GLARE 3], the improvement rate was $30.16 \%$

\section{4. the error evaluation of optimized model}

The optimized model of this study predicted the postimpact fatigue lifetime for an impact energy of $18 \mathrm{~J}$ (the maximum of very low impact energy) and a $350 \mathrm{MPa}$ tension-tension fatigue stress (the maximum exerted stress for tension-tension fatigue) as 13016 cycles. The first layer in this optimized hybrid composite was equal to GLARE 5, the second layer was equal to GLARE 3, and the third layer was equal to GLARE 5. A finite element model for this GLARE with the exact composition of the above GLARE structure was designed to validate the model with an impact energy of $18 \mathrm{~J}$ and a $350 \mathrm{MPa}$ stress level with the same finite element method whose results had good agreement with the experimental results. Afterwards, the postimpact fatigue lifetime for this new finite element model was evaluated. The obtained lifetime was 13406 cycles with a $3 \%$ difference relative to the neural network model optimized by the genetic algorithm (mean value error). Results indicated the efficacy and trustworthiness of the neural network model optimized by the genetic algorithm. The finite element model represented for this new under-the-impact GLARE composition is shown in Figure 17.

\section{Conclusion}

The purpose of the present investigation was to maximize the fatigue life of hybrid composite glass-fiberreinforced aluminum laminates (GLARE) with three permutations and optimization methods were used to find minimum values. The best configuration of hybrid composite GLARE based on conventional commercial laminates that could tolerate low-velocity impacts with
$18 \mathrm{~J}$ impact energy and the $349 \mathrm{MPa}$ fatigue load with a frequency of $10 \mathrm{~Hz}$ was [Al/0-90-90-0/Al/0-90-0/Al/090-90-0/Al] with a 13016 cycle lifetime. Given the comparison between the neural network model and the finite element model for this GLARE, the difference in the prediction of post-impact fatigue life was $3 \%$.

\section{References}

1. Sedaghat, A., Alitavoli, M., Darvizeh, A., et al. "Mathematical, numerical and experimental investigation of low energy impact on glass fiber reinforced aluminum laminates", J. of Mechanics of Continua and Mathematical Sciences, 14(3), pp. 83-93 (2019).

2. Alderliesten, R.C. "Fatigue crack propagation and delamination growth in the glare", Ph.D. Thesis, Delft University of Technology, Delft (2005).

3. Dadej, K., Surowska, B., and Bieniaś, J. "Isostrain elastoplastic model for prediction of static strength and fatigue life of fiber metal laminates", Int. J. of Fatigue, 110, pp. 31-41 (2018).

4. Park, S.Y., Choi, W.J., Choi, C.H., et al. "Effect of drilling parameters on hole quality and delamination of hybrid GLARE laminate", Composite Structures, $\mathbf{1 8 5}$, pp. 684-698 (2017).

5. Li, H., Xu, Y., Hua, X., et al. "Bending failure mechanism and flexural properties of GLARE laminates with different stacking sequences", Composite Structures, 187, pp. 354-363 (2017).

6. Zarei, H., Brugo, T., Belcari, J., et al. "Low velocity impact damage assessment of GLARE fiber-metal laminates interleaved by Nylon 6,6 nanofiber mats", Composite Structures, 167, pp. 123-131 (2017).

7. Kamocka, M., Zglinicki, M., and Mania, R.J. "Multimethod approach for FML mechanical properties prediction", Composites Part B, 91, pp. 135-143 (2016).

8. Liao, B.B. and Liu, P.F. "Finite element analysis of dynamic progressive failure properties of GLARE hybrid laminates under low-velocity impact", J. Composite Materials, 50, pp. 1-14 (2017).

9. Volt, A., Glare History of the Development of a New Aircraft Material, pp. 35-45, Dordrecht, Kluwer Academic Publishers Netherlands (2001).

10. Sadighi, M. and Alderliesten, R.C. "Impact resistance of fiber metal laminates", Int. J. of Impact Engineering, 49, pp. 77-90 (2012).

11. Wu, G.C. and Yang, J.M. "The mechanical behavior of GLARE laminates for aircraft structures", J Minerals Metals Mater, 57, pp. 72-79 (2005).

12. Liang, Z.Q. and Xue, Y.D. "Performance and application of GLARE laminates in A380 Airliner. Glass FRP/CM", Int. J. Composite, 04, pp. 49-51 (2005). 
13. Liang, Z.Q. and Wu, W.J. "Comparison of GLARE laminates with aluminum alloy and its application", J. of the Minerals, Metals \& Materials Society, 57(1), pp. $72-79$ (2006).

14. Syed, A.K., Zhang, X., Moffatt, J.E., et al. "Fatigue performance of bonded crack retarders in the presence of cold worked holes and interference-fit fasteners", Int. J. of Fatigue, 105, pp. 111-118 (2017).

15. Al-Azzawi, A.S., Mc Crory, J., Kawashita, L.F., et al. "Buckling and postbuckling behaviour of glare laminates containing splices and doublers. Part 1: Instrumented tests", Composite Structures, 176, pp. 1158-1169 (2017).

16. Wang, W., Rans, C., and Benedictus, R. "Analytical prediction model for non-symmetric fatigue crack growth in fibre metal laminates", Int. J. of Fatigue, 103, pp. 546-556 (2017).

17. Marissen, R. "Fatigue crack growth in ARALL, a hybrid aluminium-aramid composite material, crack growth mechanisms and quantitative predictions of the crack growth rate", Dissertation for the Doctoral Degree, Delft University of Technology (1988).

18. Lapczyk, I. and Hurtado, J.A. "Progressive damage modeling in fiber-reinforced materials", Composites Part A, 38, pp. 2333-2341 (2007).

19. Graupe, D. and Kordilewski, H. "A novel largememory neural network as an aid in medical diagnosis", IEEE Trans. on Information Technology in Biomedicine, 5(3), pp. 202-209 (2001).

20. Hagan, M.T. and Menhaj M.B. "Training feedforward networks with the Marquardt algorithm", IEEE Trans. on Neural Networks, 5, pp. 989-993 (1994).

21. Azadeh, A., Saberi, M., TavakkoliMoghadam, R., and Javanmardi, L. "An integrated Data envelopment analysis-artificial neural network-rough set algorithm for assessment of personnel efficiency", Expert Systems with Applications, 38, pp. 1364-1373 (2011).

22. Haykin, S., Neural Networks: A Comprehensive Foundation, pp. 134-168, Prentice Hall, Dehli, India (1990).

23. Lei, Y., He, Z., and Zi, Y. "Application of an intelligent classification method to mechanical fault diagnosis", Expert Systems with Applications, 36, pp. 9941-9948 (2009).

24. Li, B., Chow, M.Y., Tipsuwan, Y., et al. "The neuralnetwork-based motor rolling bearing fault diagnosis", IEEE Tran. on Industrial Electronics, 47, pp. 10601069 (2000).
25. Soutis, C., Mohamed, G., and Hodzic, A. "Modelling the structural response of GLARE panels to blast load", Composite Structures, 94, pp. 267-276 (2011).

26. Donadon, M.V., Iannucci, L., Falzon, B., et al. "A progressive failure model for composite laminates subjected to low-velocity impact damage", Composite Structures, 86(12), pp. 1232-1252 (2008).

27. Apruzzese, P. and Falzon B. "Numerical analysis of complex failure mechanisms in composite panels", 16th International Conference on Composite Materials, Kyoto, Japan, pp. 234-246 (2007).

28. Wang, W., Rans, C., Zhang, Z., and Benedictus, R. "Prediction methodology for fatigue crack growth behaviour in fibre metal laminates subjected to tension and pin loading", Composite Structures, 185, pp. 176$182(2017)$.

29. Seyed Yaghoubi, A. and Liaw, B. "Thickness influence on ballistic impact behaviors of GLARE 5 fiber-metal laminated beams: Experimental and numerical studies", Composite Structures, 94, pp. 2585-2598 (2012).

\section{Biographies}

Alireza Sedaghat is a $\mathrm{PhD}$ student of Mechanical Engineering at Guilan University and Faculty Member of Islamic Azad University of Lahijan. His main research interests include: impact mechanics, fatigue, hybrid composites, and biomechanics.

Majid Alitavoli is an Associate Professor at the Faculty of Mechanical Engineering of Guilan University. His main research interests include metal forming, water jet, innovative design, and impact.

Abolfazl Darvizeh is a distinguished Professor in Mechanical Engineering at Guilan University. His main research interests include design and nature (biomimetic engineering), impact mechanics, and explosive welding.

Reza Ansari Khalkhali is a Full Professor at the Faculty of Mechanical Engineering of Guilan University. His main research interests include mathematical modeling, dynamics and vibrations, and non-conventional materials. 